\title{
Tendências temporais da morbidade e da mortalidade por doenças respiratórias na cidade de São Paulo*
}

\section{Temporal trends in morbidity and mortality for respiratory diseases in São Paulo, Brazil}

\author{
Marcos Tadashi Kakitani Toyoshima(1), Gláucia Munemasa Ito(1), \\ Nelson Gouveia(2)
}

\begin{abstract}
Toyoshima MTK, Munemasa G, Gouveia N. Tendências temporais da morbidade e da mortalidade por doenças respiratórias na cidade de São Paulo. Rev Med (São Paulo) 2003 jan.-dez.;82(1-4):67-77.

RESUMO: Doenças respiratórias são importante causa de morbi-mortalidade em todas as faixas etárias, com considerável impacto econômico e social. Diversos autores vêm apontando um aumento nas hospitalizações e mortes por algumas causas respiratórias, porém em nosso meio, poucos estudos analisaram estas tendências recentemente. No presente trabalho, dados de hospitalizações para o período de 1995 a 2000 e de mortalidade para 1991-2000 foram analisados através de tabelas e gráficos descritivos, testes de correlação e de tendência linear. Observou-se que houve decréscimo na taxa de internações por doenças respiratórias entre 1995-1997 e tendência de aumento de 1997-2000 em todas as idades. Tendência crescente foi também observada na mortalidade por doenças respiratórias entre 1991-2000. Padrões específicos para diferentes grupos de doenças respiratórias, diferentes faixas etárias e sexos foram observados. Concluímos que no período estudado, houve participação importante na morbi-mortalidade respiratória de doenças agudas, como as pneumonias, mas com tendência a diminuir a sua participação, ao contrário de doenças crônicas como a DPOC. Estes resultados indicam uma mudança no perfil destas doenças, o que traz consequiências imediatas para a formulação de políticas de saúde.
\end{abstract}

DESCRITORES: Perfil de impacto da doença. Comorbilidade. Doenças/mortalidade. Indicadores de morbi-mortalidade. Variações sazonais. Fatores de tempo.

\section{INTRODUÇÃO}

As doenças respiratórias constituem importante causa de morbi-mortalidade, mas se observa que a maioria dos projetos de pesquisa se concentra em países desenvolvidos, cujos dados não são exatamente adequados para aplicação na nossa realidade ${ }^{14,15}$. No Brasil, poucos estudos procuraram analisar o perfil de morbi-mortalidade por doenças respiratórias nas grandes cidades, e menos ainda as tendências temporais recentes.

\footnotetext{
* Vencedor do Prêmio Oswaldo Cruz, área - Medicina Preventiva, XXI COMU 2002.

(1) Acadêmicos da Faculdade de Medicina da Universidade de São Paulo

(2) Orientador, Prof. Depto. de Medicina Preventiva da Faculdade de Medicina da Universidade de São Paulo

Endereço para correspondência: Marcos T. K. Toyoshima. Rua: Professor Antônio Prudente, 41 Apto. 506, São Paulo, SP, Brasil. CEP: 01509-010. e-mail: mtkt@terra.com.br
} 
A asma, por exemplo, é importante causa de morbidade associada a prejuízos financeiros e psicológicos a longo prazo, e a grande utilização dos serviços de saúde, sendo incomum causa de mortalidade, uma vez que é tratável e controlável ${ }^{1}$. Mesmo assim, alguns países vêm reportando aumento na mortalidade por asma ${ }^{3,4}$.

As pneumonias são a sexta causa mais importante de morte nos EUA e são o principal diagnóstico de infecção respiratória aguda ${ }^{13,33}$ A taxa de mortalidade vem caindo ao longo dos anos, nos EUA, exceto entre os idosos ${ }^{15}$, fato que reflete tanto o crescimento da população idosa quanto também o maior acometimento dessa faixa etária ${ }^{24}$.

Tendo em vista a importância da morbi-mortalidade por doenças respiratórias agudas e crônicas e as indicações de que vêm aumentando nos últimos anos, este estudo pretendeu descrever a evolução das doenças respiratórias em geral, por pneumonias (principal diagnóstico entre infecções respiratórias agudas) ${ }^{8}$, por asma e por DPOC (representantes das doenças respiratórias crônicas) ${ }^{9}$ e analisar as tendências de morbidade no Município de São Paulo de 1995 a 2000 de mortalidade de 1991 a 2000.

\section{MATERIAIS E MÉTODOS}

\section{a) Informações de morbidade e de mortalidade}

As informações referentes às internações hospitalares, para todas as idades e causas, de residentes do Município de São Paulo, foram obtidas através das Autorizações de Internações Hospitalares (AIH) do SUS - Ministério da Saúde, disponíveis em CD-ROMs. Estes bancos de dados contêm informações de todas as internações realizadas no âmbito do SUS através das AIH como sexo, idade, data de internação, data de alta, diagnóstico, duração da internação, identificação do hospital, unidade da federação, entre outras. Apesar de ser um instrumento administrativo, alguns estudos têm demonstrado a confiabilidade dos dados da AIH para estudos epidemiológicos ${ }^{9}$.

Os dados de mortalidade foram fornecidos pelo Programa de Aperfeiçoamento das Informações de Mortalidade (PROAIM) ${ }^{30}$ da Prefeitura do Município de São Paulo, que analisa e processa as informações contidas nos atestados de óbitos ocorridos dentro do município, cujos dados contêm informações como data do óbito, sexo, idade, endereço residencial, onde ocorreu a morte e causa básica de óbito codificada de acordo com a Classificação Internacional da Doença (CID). O uso de atestados de óbitos como instrumento para estudos epidemiológicos teve seu uso validado na cidade de São Paulo em dois estudos e, quando comparado com outras cidades, é considerado confiável ${ }^{22}$.

\section{b) Formatação dos bancos de dados}

Os dados obtidos vieram no formato dBASE e foram abertos como planilhas do Microsoft ${ }^{\circledR}$ Excel 2000.
Como o número de internações para cada ano era contabilizado a partir da data de saída do hospital, foi necessária adaptação dos dados através da consideração da data de internação como parâmetro de internação em cada ano.

Utilizaram-se os capítulos VIII e X, da $9^{\mathrm{a}}$ e $10^{\mathrm{a}}$ Classificação Internacional de Doenças. Até 1997, os dados de doenças eram disponíveis de acordo com a CID9. Em 1998, foram utilizadas ambas classificações e a partir de 1999, somente a CID-10. Observamos as tendências temporais de grupos de doenças pulmonares, como pneumonias, as doenças pulmonares obstrutivas crônicas (DPOC), a asma e a gripe, utilizando-se os códigos de diagnósticos da CID-9 e CID-10 $26,27$.

Em destaque, separamos duas populações de faixas etárias, denominando-as como faixas etárias I e III, representadas, respectivamente, pelos pacientes com idade menor de cinco anos e pelos pacientes com idade maior de 64 anos, comparando com a faixa etária intermediária (faixa etária II).

\section{c) Análise epidemiológica dos dados}

Para a análise de tendência linear foram utilizados regressão linear e teste de correlação de Pearson. Para a comparação de médias, utilizou-se análise de variância (ANOVA). Lançamos mão do Epi Info versão 6.04b. Como seria mais conveniente trabalhar com taxas de internações e de mortalidade, estimativas da população total e para cada sexo foram obtidas da Fundação Sistema Estadual de Análise de Dados Estatísticos (SEADE) e do DATASUS ${ }^{12,34}$.

\section{RESULTADOS}

\section{Dados de morbidade}

\section{A. Doenças respiratórias}

Através das AIHs contabilizamos 206764 internações por doenças respiratórias no município de São Paulo no período de 1995 a 2000. No evoluir desses anos, houve diminuição percentual de $22 \%$ no número absoluto de hospitalizações. Quanto às taxas de internações, em 1995, eram 41,7 internações por 10 mil habitantes passando a 31,1 em 2000, o que representa redução de $26 \%$. Todavia, apesar da análise da tendência geral no período ter sido de decréscimo na taxa de internações $(r=-0,74)$, a mesma não foi estatisticamente significante $(\mathrm{p}=0,09)$ (Gráfico 1).

Comparando a evolução das doenças respiratórias para as populações masculina e feminina, observamos que a taxa de internações foi maior em homens do que em mulheres em todos os anos estudados. No período de 1995 a 2000, houve, em linhas gerais, decréscimo da taxa em ambos os sexos. Somente para os homens, houve queda significante, sendo o valor de $\mathrm{r}=-0,85(\mathrm{p}=0,03)$. Para o sexo feminino, $r=-0,49(\mathrm{p}=0,32)$ (Gráfico 2). 


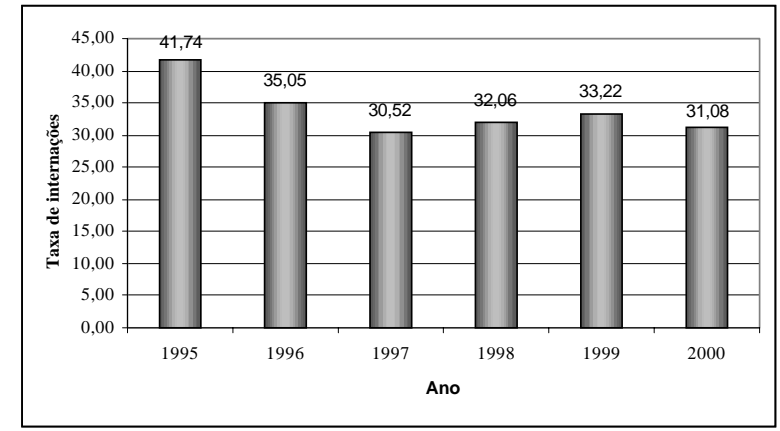

Gráfico 1. Evolução da taxa de internações (por 10 mil habitantes) das doenças respiratórias no Município de São Paulo (1995-2000)

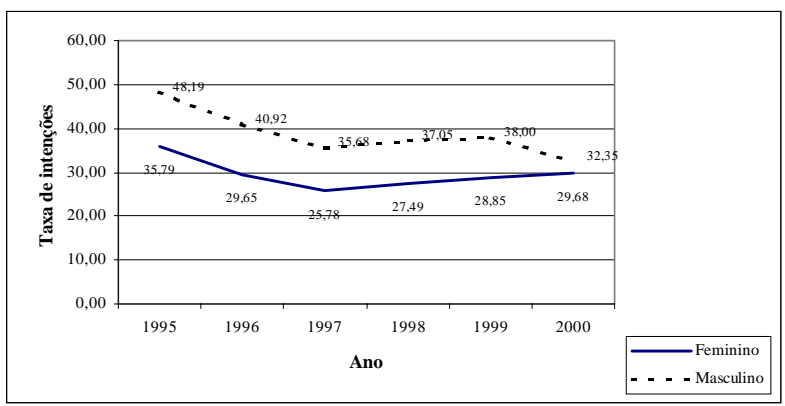

Gráfico 2. Evolução por sexo da taxa de internações por 10 mil habitantes das doenças respiratórias no Município de São Paulo (1995-2000)

Analisando os três grupos de faixas etárias, observamos maior taxa de internações na faixa etária I, seguida da III. Observamos nas três, quedas da taxa, mas em nenhuma delas houve diminuição significante, sendo obtidos $r=-0,39(p=0,32)$ para a faixa etária $I, r=-0,40$ $(\mathrm{p}=0,44)$ para a faixa II e $\mathrm{r}=-0,73(\mathrm{p}=0,10)$ para a III (Gráfico 3).

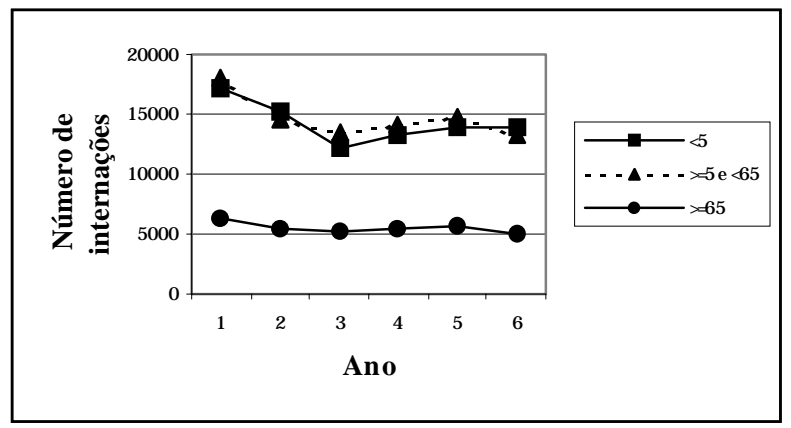

Gráfico 3. Evolução do número de internações por doenças respiratórias no Município de São Paulo, conforme idade (1995-2000)

Na análise da existência de um padrão sazonal no número de internações por doenças respiratórias, observamos a mesma evolução em todos os anos: aumento do número de internações no primeiro semestre e diminuição no segundo. Os picos sempre se encontravam entre os meses de abril a junho (mais relacionados ao período de inverno) e os vales, entre dezembro a fevereiro (mais relacionados ao verão). Pela análise de variância, há padrão sazonal no total de doenças respiratórias com dados significativos $(\mathrm{p}<0,01)$ (Gráfico 4).

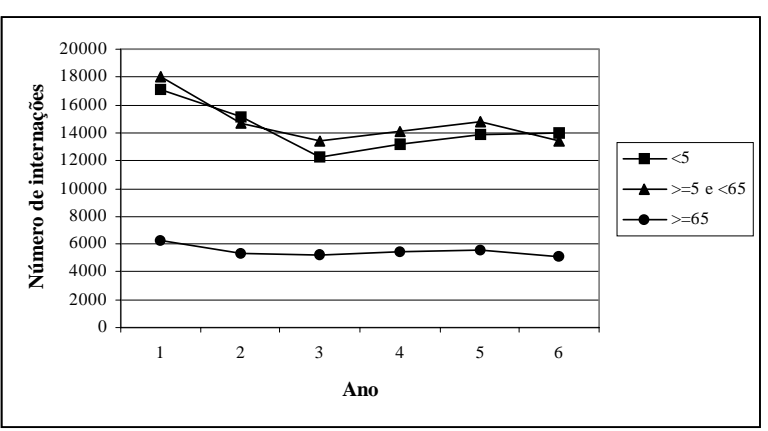

Gráfico 4. Evolução do número de internações por doenças respiratórias no Município de São Paulo (1995-2000)

\section{respiratórias}

\section{B. Comparação entre os grupos de doenças}

Para o período estudado como um todo, houve grande contribuição das pneumonias no total de internações por doenças respiratórias, correspondendo a $47 \%$. Todavia, observamos aumento da contribuição no número de internações da DPOC e da asma no período de 1995 a 2000, com conseqüente diminuição no grupo das pneumonias (Gráfico 5).

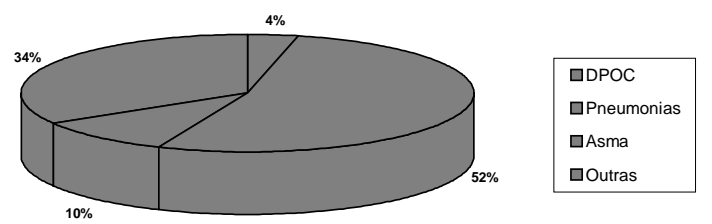

Gráfico 5. Distribuição das doenças respiratórias por número de internações no Município de São Paulo no período de 1995 a 2000

A média de idades dos pacientes internados foi de 57,7 anos para DPOC, 23,8 anos para pneumonias e 19,0 anos para asma. Houve aumento da média de idades dos pacientes internados por DPOC, sendo de 50,7 anos em 1995 e 61,9 anos em 2000. Para a asma e para as pneumonias, as médias se mantiveram.

Quanto ao tempo de hospitalização, para a DPOC no período de 1995 a 2000 evoluiu de 7,96 dias para 11,24 dias. Para pneumonias, asma e para o total de doenças respiratórias, o tempo de hospitalização se manteve.

\section{Doenças respiratórias específicas}

Cada um dos três grupos de doenças pulmonares já citados foi analisado separadamente. 

(DPOC)

a) Doenças pulmonares obstrutivas crônicas

Notamos aumento do número de internações em geral, em cada faixa etária e principalmente, para a faixa etária III, no período de 1995 a 2000.

A tendência geral observada da taxa de internações por DPOC no período foi de aumento $(r=0,75)$, com resultado não estatisticamente significante $(\mathrm{p}=0,08)$. Em 1995, notamos que a taxa de internações foi de 15,64 internações/100 mil habitantes e máximo de 27,14, no ano de 1998 , o que representa um aumento de $73,5 \%$ nesse período.

A taxa de internações foi maior na população masculina do que na feminina nos anos estudados, com tendência de aumento para ambos. Para o sexo masculino, obtivemos o valor de $\mathrm{r}=0,66$, mas com aumento não estatisticamente significante $(\mathrm{p}=0,15)$. Entretanto, foi observado aumento estatisticamente significante para o sexo feminino $(r=0,82$ e $p=0,04)$.

Comparando os três grupos de faixas etárias, observamos que as faixas II e III contribuíram com o maior número absoluto de internações em todos os anos.

Em taxa de internações, a faixa etária III obteve maior taxa de internações por DPOC, com aumento abrupto em 1998 desta faixa etária. Em 1995, a taxa de internações era de 6,94 internações/10 mil habitantes maiores de 60 anos, passando a 14,53 no ano de 2000. Assim sendo, o valor do $r$ foi de $0,84(p=0,03)$ (Gráfico 6).

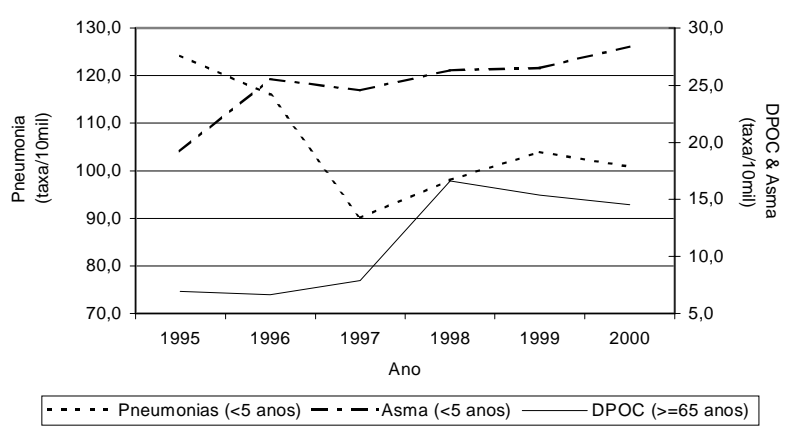

Gráfico 6. Evolução da taxa de internações (por $10 \mathrm{mil}$ habitantes) para doenças respiratórias e faixas etárias específicas no Município de São Paulo (1995-2000)

Para as outras faixas etárias, obtivemos diminuição da taxa de internações no período para a faixa I, sendo $r=-0,73$ e $p=0,10$; e aumento para a II, com $p=0,68$ e $\mathrm{p}=0,13$.

Maior número de internações ocorreu entre os meses de maio a julho (mais relacionados ao inverno) e menor, entre novembro e janeiro (mais relacionados ao verão). Essa variação mostrou-se não estatisticamente significativa $(\mathrm{p}=0,23)$ pela ANOVA.

\section{b) Asma}

Não houve alteração importante do número de internações por asma no período de 1995 a 2000; o mesmo com as taxas de internações, sendo o cálculo de $\mathrm{r}=0,14$, com $\mathrm{p}=0,79$. Em 1995, obtivemos taxa de 4,20 internações por 10 mil habitantes e de 4,24 em 2000.

$\mathrm{Na}$ análise das taxas de internações por asma em cada sexo, observamos que foi maior na população masculina do que na feminina de 1996 a 1999. Em 1995 e 2000 (o primeiro e o último ano estudado, respectivamente), tal situação se inverteu.

Em linhas gerais, observamos tendências diferentes na taxa de internações entre os sexos. Para o sexo masculino, houve pequeno aumento da taxa de internações $(\mathrm{r}=0,23)$ e para o feminino, ausência de tendência $(r=-0,02)$. Ambas situações não foram significantes.

Em termos de número de internações, notamos para a faixa etária I um discreto aumento do número de casos de 1995 a 1999. Já para a faixa II, houve oscilação do número de internações, com diminuição de 1995 a 1998 e aumento de 1998 a 1999. E finalmente, para a faixa III foi observado número estável de internações. Para as taxas de internações, houve tendência de aumento no período apenas para a faixa etária $\mathrm{I}$, sendo $\mathrm{r}=0,86$. Para as faixas II e III, diminuição das taxas, sendo, respectivamente, valores de $\mathrm{r}$ iguais a $-0,49 \mathrm{e}-0,26$. Somente as internações de pacientes com idade menor de 5 anos obtiveram alteração estatisticamente significante de 1995 a 2000 (Gráfico 6).

Quanto à existência de um padrão sazonal na asma, observamos flutuação no número de internações no decorrer dos meses, com máximo entre março e maio e mínimo em janeiro. Pela análise de variância, a variação é estatisticamente significativa, com $\mathrm{p}<0,01$.

\section{c) Pneumonias}

Observamos redução no número absoluto de internações por pneumonias no período de 1995 a 2000. Quanto às taxas de internações, notamos redução de 1995 a $2000(r=-0,73, p=0,10)$. De 1995 a 1997, observamos diminuição das taxas de internações $(21,93$ internações por 10 mil habitantes em 1995 e 13,49, em 1997). De 1997 a 1999, aumento das taxas, seguido de diminuição em 2000, com taxa de internação igual a 14,0.

Quanto ao sexo dos pacientes, observamos maior taxa de internações na população masculina do que na feminina em todos os anos estudados. De 1995 a 2000, a diferença de taxas entre os sexos decaiu de 7,5, em 1995, para 1,3, em 2000. Houve diminuição das taxas de internações no período de 1995 a 2000 para ambos os sexos; porém, mais acentuada na população masculina ( $\mathrm{r}$ $=-0,81 \mathrm{p}=0,05)$ do que na feminina $(\mathrm{r}=-0,57 \mathrm{p}=0,23)$.

$\mathrm{Na}$ análise dos três grupos de faixas etárias, observamos maior número absoluto de internações por pneumonias na faixa etária I, seguida pela faixa II e por último, a III. Para as faixas etárias I e II, houve diminuição 
do número de internações no período de 1995 a 1997 e discreto aumento, a partir de 1997. Para a faixa etária III, houve diminuição de 1995 a 1996 e manutenção do número de casos nos anos seguintes.

Observamos maiores taxas de internações por pneumonias na faixa etária I em todos os anos estudados. Notamos que as faixas etárias extremas possuem taxas maiores de internação do que a intermediária. Foi observado anteriormente, que em número de internações a faixa etária II superava a III. Tendo em vista que aquela faixa etária é mais populosa, as taxas de internações representam melhor a participação das pneumonias nas três faixas etárias. Com a tendência de envelhecimento e maior expectativa de vida da população, espera-se maior participação das pneumonias na faixa etária III também em números absolutos.

Nas três faixas estudadas, houve redução das taxas de internações, com resultados significantes. Os valores encontrados foram: $r=-0,62(p=0,19) ; r=-0,73$ $(\mathrm{p}=0,10) ; \mathrm{r}=-0,63(\mathrm{p}=0,17) ;$ respectivamente, para as faixas etárias I, II e III (Gráfico 6).

Houve aumento do número de internações no primeiro semestre e queda, no decorrer do segundo. Os meses com maior número de internações são os mais relacionados ao inverno (abril a julho) e aqueles com menor número são os mais relacionados com o verão (novembro a fevereiro). Pela ANOVA, há padrão sazonal, com dados significativos $(\mathrm{p}<0,01)$.

\section{Dados de mortalidade}

\section{A. Doenças respiratórias}

As taxas de mortalidade por 10000 habitantes para doenças respiratórias totais apresentam decréscimo $(\mathrm{r}=-0,63)$, porém não muito significante $(\mathrm{p}=0,0541)$ no período estudado.

Comparando-se a taxa de mortalidade entre homens e mulheres percebe-se o aumento de ambas as taxas para o período de 1991 para 1994, seguida por tendência à queda a partir de 1994, mantendo certa estabilidade a partir de 1996. A população masculina é a mais acometida em todos os anos. Em relação ao total de casos, 56\% são homens (Gráfico 1). Observa-se um decréscimo real para homens $(r=-0,63, p=0,05)$, não havendo uma diminuição significante entre as mulheres $(r=-0,28, p=0,43)$ ao longo do período.

A mortalidade por doenças respiratórias é maior na faixa etária III e em seguida, a I. Mesmo levando-se em conta que a faixa etária II é muito abrangente, a sua importância é a menor, com as menores taxas (Tabela 1).

Tabela 1. Taxa de mortalidade de acordo com faixa etária em cada sexo, no município de São Paulo, por 10000 habitantes (19912000)

\begin{tabular}{|c|c|c|c|c|c|c|c|c|c|c|}
\hline Masculino & 1991 & 1992 & 1993 & 1994 & 1995 & 1996 & 1997 & 1998 & 1999 & 2000 \\
\hline Menores que 5 anos & 12.250 & 12.453 & 13.662 & 12.531 & 11.946 & 11.377 & 9.475 & 7.516 & 6.235 & 5.331 \\
\hline Entre 5 e 65 & 3.801 & 3.881 & 4.304 & 4.338 & 4.147 & 3.277 & 2.713 & 2.819 & 2.601 & 2.679 \\
\hline Maiores que 65 anos & 53.776 & 62.287 & 72.211 & 72.653 & 71.445 & 60.753 & 59.659 & 57.982 & 61.059 & 62.794 \\
\hline Total & 7.563 & 8.830 & 9.961 & 9.911 & 9.610 & 8.266 & 7.548 & 7.343 & 7.281 & 7.398 \\
\hline Feminino & 1991 & 1992 & 1993 & 1994 & 1995 & 1996 & 1997 & 1998 & 1999 & 2000 \\
\hline Menores que 5 anos & 9.554 & 9.905 & 11.777 & 10.718 & 9.932 & 8.760 & 6.222 & 7.082 & 5.610 & 4.604 \\
\hline Entre 5 e 65 & 1.638 & 1.816 & 2.088 & 2.005 & 1.973 & 1.574 & 1.596 & 1.588 & 1.628 & 1.514 \\
\hline Maiores que 65 anos & 33.995 & 38.190 & 45.961 & 49.718 & 46.322 & 42.706 & 41.793 & 40.012 & 43.442 & 43.937 \\
\hline Total & 5.267 & 5.854 & 6.917 & 7.096 & 6.693 & 6.136 & 5.871 & 5.757 & 6.011 & 5.888 \\
\hline
\end{tabular}

Nota-se decréscimo na faixa etária II $(r=-0,79)$ e na $\mathrm{I}(\mathrm{r}=-0,90)$ ao longo do período com resultados estatisticamente significantes $(\mathrm{p}=0.05)$. Na faixa etária III, não há uma tendência clara $(\mathrm{r}=0,06 \mathrm{p}=0,86)$.

As taxas de mortalidade são mais elevadas entre os homens atingindo todas as faixas etárias. Na faixa etária
III, permanecem em média 63 por 10000 habitantes homens enquanto para mulheres, atingem 42 por 10000 habitantes. As outras faixas etárias mostram distribuição menos discrepante entre homens e mulheres, em média, taxa de 8,5 por 10000 habitantes para a faixa etária I em mulheres e de 10 por 10000 habitantes, para homens; para 
a faixa etária II, taxas de 1,7 para mulheres e de 3,5 para homens.

\section{B. Comparação entre os grupos de doenças respiratórias}

A Tabela 2 mostra a evolução do número de mortes ocorridas no período de 1991 a 2000 pelas doenças respiratórias, mostrando que a causa de morte mais importante entre as doenças respiratórias é a pneumonia sendo em média responsável por $61 \%$ dos óbitos por doença respiratória (Gráfico 2). A asma, a pneumonia e a gripe tiveram diminuição do número de casos de 1995 para 1996, talvez correlacionadas com a mudança da CID. A reclassificação tem algum impacto nas taxas refletindo a mudança no primeiro ano logo após a modificação. A manutenção de níveis de valores provavelmente é a real tendência das curvas ${ }^{37}$.

Tabela 2. Números absolutos de morte por doença respiratória, no município de São Paulo (1991-2000)

\begin{tabular}{|c|c|c|c|c|c|c|c|c|c|c|c|c|}
\hline & 1991 & 1992 & 1993 & 1994 & 1995 & 1996 & 1997 & 1998 & 1999 & 2000 & Total & $\%$ \\
\hline DPOC & 1675 & 1777 & 1973 & 2075 & 2199 & 2542 & 2512 & 2403 & 2618 & 2623 & 22397 & 35,93 \\
\hline Asma & 105 & 173 & 204 & 212 & 214 & 186 & 180 & 170 & 185 & 198 & 1827 & 2,93 \\
\hline Pneumonias & 3701 & 4112 & 4939 & 4993 & 4941 & 3558 & 3052 & 3022 & 2865 & 2884 & 38067 & 61,07 \\
\hline Doenças respiratórias & 6433 & 7081 & 8287 & 8494 & 8267 & 7021 & 6576 & 6450 & 6581 & 6605 & 71795 & 100.00 \\
\hline
\end{tabular}

Como os casos de gripe apareceram em quantidade irrisória neste período, esse grupo de doença foi excluído de nossas análises.

A Tabela 3 mostra a contribuição relativa de cada doença respiratória específica para as faixas etárias aqui estudadas. Percebe-se que as pneumonias representam a maior causa de morte entre as doenças respiratórias, em especial para os menores que cinco anos de idade, representando cerca de $80 \%$ das mortes respiratórias. DPOC tem sua importância crescente a partir da faixa etária intermediária e aumenta entre os idosos. Em números absolutos, a asma tem maior importância na faixa etária II. As outras doenças respiratórias têm distribuição quase constante entre as três faixas etárias.

Tabela 3. Doenças respiratórias por faixa etária

\begin{tabular}{|c|c|c|c|c|c|c|c|c|}
\hline & $<5$ anos & $\%$ & entre 5-65 & $\%$ & ${ }^{3} 65$ anos & $\%$ & Total & $\%$ \\
\hline Asma & 64 & 0.79 & 1024 & 4.85 & 736 & 1.73 & 1824 & 2.55 \\
\hline Pneumonias & 6460 & 79.84 & 11238 & 53.24 & 20263 & 47.73 & 37961 & 52.98 \\
\hline DPOC & 203 & 2.51 & 5671 & 26.87 & 16506 & 38.88 & 22380 & 31.23 \\
\hline Outras & 1364 & 16.86 & 3176 & 15.05 & 4948 & 11.66 & 9488 & 13.24 \\
\hline Doenças respiratórias & 8091 & 100.00 & 21109 & 100.00 & 42453 & 100.00 & 71653 & 100.00 \\
\hline
\end{tabular}

De maneira geral, a taxa de mortalidade por 10000 habitantes foi crescente para DPOC, pneumonia e asma na primeira metade do período estudado. A asma apresentou o mesmo padrão de diminuição de 1995 para 1996 (Tabela 2), enquanto a pneumonia manteve o decréscimo nos anos de 1994 para 2000. As Pneumonias apresentaram decréscimo $(\mathrm{r}=-0,73)$ estatisticamente significante $(\mathrm{p}=0,05)$. DPOC apresentou crescimento $(\mathrm{r}=0,93)$, também significante $(p=0,05)$ enquanto asma apontou crescimento $(r=0,23)$, porém não estatisticamente significante $(\mathrm{p}>0,05)$.

\section{Doenças respiratórias específicas}

\section{a) DPOC}

Os mais acometidos são os idosos sendo seguidos pela faixa intermediária. As taxas para DPOC têm tendência claramente crescente nessas duas faixas etárias. Para a faixa etária $\mathrm{I}, \mathrm{r}=0,15$, porém $\mathrm{p}>0,05$, enquanto para a faixa II $(r=0,95)$ e III $(r=0,93)$, o crescimento foi estatisticamente significante $(\mathrm{p}=0,05)$, coincidindo com o observado no Gráfico 3.

Em média, entre os menores que 5 anos, a taxa para homens é de 0,28 e para mulheres de 0,20 . Na faixa II, é de 0,80 para homens e 0,60 para mulheres, e para a III, a média foi de 29,00 para homens e 13,50 para mulheres. Estatisticamente, nota-se crescimento de taxas tanto para homens $(\mathrm{R}=0,92)$, quanto para mulheres $(\mathrm{R}=0,93)$ com $\mathrm{p}=0,05$ ao longo do período.

\section{b) Asma}

O acometimento da faixa etária III foi maior, sendo seguido pela faixa II, como ocorre na DPOC. Na faixa 
etária I, houve padrão decrescente $(r=-0,09)$, sendo que na faixa etária II $(r=0,32)$ e III $(r=0,21)$ houve crescimento, porém nenhum estatisticamente significante $(\mathrm{p}>0,05)$.

A taxa de mortalidade por 10000 habitantes para cada sexo e faixa etária mostra valores próximos entre homens e mulheres. Entretanto, diferentemente das outras doenças, as mulheres (taxa média de 0,22/10000) são mais acometidas que os homens (0,14/10000). Entre os menores que 5 anos, uma média de 0,088 para homens e 0,062 para mulheres. Na faixa intermediária, 0,093 para homens e 0,154 para mulheres; e nos idosos, 0,792 para homens e 0,955 para mulheres (taxa maior para mulheres nas duas faixas etárias que incluem os adultos).

Os homens apresentaram decréscimo $(r=-0,08)$ e as mulheres, crescimento $(\mathrm{r}=0,40)$, porém não significantes $(\mathrm{p}>0,05)$ ao longo do período.

\section{c) Pneumonias}

As pneumonias são as causas de morte mais importantes entre as doenças respiratórias e exercem influência sobre a curva de doenças respiratórias no total. As tendências decrescentes de pneumonias (Tabela 4) observadas neste estudo foram relatadas em outros estudos ${ }^{22}$. Possíveis fatores que explicam essa tendência podem estar relacionadas com melhor oferta de tratamento ${ }^{38}$.

Tabela 4. Taxa de mortalidade por doenças respiratórias, para grupos de causas, MSP, por 10000 habitantes 1991-2000

\begin{tabular}{|c|c|c|c|c|c|c|c|c|c|c|}
\hline & 1991 & 1992 & 1993 & 1994 & 1995 & 1996 & 1997 & 1998 & 1999 & 2000 \\
\hline DPOC & 1.74 & 1.83 & 2.02 & 2.11 & 2.21 & 2.54 & 2.48 & 2.35 & 2.54 & 2.52 \\
\hline Asma & 0.11 & 0.18 & 0.21 & 0.22 & 0.22 & 0.19 & 0.18 & 0.17 & 0.18 & 0.19 \\
\hline Pneumonias & 3.85 & 4.24 & 5.06 & 5.07 & 4.97 & 3.55 & 3.02 & 2.96 & 2.78 & 2.77 \\
\hline Doenças respiratórias & 6.69 & 7.31 & 8.48 & 8.62 & 8.32 & 7.00 & 6.50 & 6.32 & 6.39 & 5.49 \\
\hline
\end{tabular}

De acordo com o observado no Gráfico 4, a mortalidade por pneumonias atinge principalmente os mais idosos, sendo seguidos pelos menores que 5 anos, diferentemente das outras doenças que atingem em segundo lugar a faixa etária intermediária. Isso se reflete no total de doenças respiratórias, pela grande importância das pneumonias. Percebe-se um padrão decrescente para a taxa de mortalidade por pneumonias por todas as faixas etárias, havendo um crescimento entre os mais idosos no período de 1991 a 1994, com queda importante entre 1995 e 1996, ano da mudança da CID, porém mantendo-se nos mesmos níveis nos anos seguintes. As taxas de mortalidade por faixas etárias e por sexo mostraram evolução semelhante.

Os menores que 5 anos e a faixa intermediária tiveram decréscimo ao longo do período (respectivamente $r=-0,88$ e $r=-0,84, p=0,05)$. Os idosos apresentaram tendência à queda $(\mathrm{r}=-0,47)$, porém não significante $(\mathrm{p}>0,05)$.

Os homens menores que 5 anos têm taxa de 8,2 em média, e as mulheres, 6,6 por 10000 habitantes. Na faixa etária II, os homens ficam em torno de 2,0 e as mulheres de 0,8 . Entre os idosos, em média, 27 para homens e 23 para mulheres.

Durante o período, percebemos decréscimo tanto para homens $(\mathrm{r}=-0,80, \mathrm{p}=0,05)$ quanto para mulheres $(r=-0,60, p>0,05)$, embora, neste caso, não sendo significante.

\section{d) Padrões sazonais}

A mortalidade por DPOC apresenta caráter oscilatório e gráfico crescente. A asma também apresenta a oscilação parecendo ter leve aumento ao longo do período apesar da grande variabilidade. A curva das pneumonias vem diminuindo ao longo do tempo, principalmente a partir de 1995. Os números permanecem altos durante os meses de verão, apresentando importante aumento nos meses de inverno.

A evolução das doenças respiratórias ao longo do período mostra que o número de casos de morte aumenta durante os meses de inverno e diminui nos meses de verão. Doenças respiratórias totais mostram variação durante os meses do ano, crescimento das taxas durante o inverno e queda no verão, sendo estatisticamente significante (Kruskal-Wallis; $\mathrm{p}=0,05$ ). DPOC não mostra variação estatisticamente significante em nenhum teste. Enquanto as pneumonias mostram variação estatisticamente significante $(\mathrm{p}=0,05)$ por ANOVA. A asma também mostra variação, com $\mathrm{p}=0,05$, pelo teste de KruskalWallis.

\section{DISCUSSÃO}

As doenças respiratórias constituem uma das principais causas de enfermidade e morte em adultos e crianças $^{15}$. Segundo dados da Organização Mundial de Saúde (OMS), as doenças do sistema respiratório representaram $8 \%$ do total de mortes em países desenvolvidos e $5 \%$ em países em desenvolvimento, no ano de $1997^{40}$.

Nos países em desenvolvimento, a OMS estima que em 1995 4,3 milhões de crianças com idade inferior a cinco anos morreram por doenças respiratórias agudas, sendo a principal causa as pneumonias. Quanto à morbidade, estima-se que cerca de 40 milhões de crianças menores de 
cinco anos adquirem pneumonia anualmente ${ }^{39}$.

Em nosso trabalho, tal doença correspondeu a aproximadamente $47 \%$ do número de internações por doenças respiratórias no período estudado (Gráfico 5) Convém salientarmos que o número de internações de cada doença não reflete nem a prevalência tampouco a incidência dessas morbidades na população. Crises leves de doença respiratória podem ter controle domiciliar desde que os pacientes sejam bem instruídos no manejo terapêutico. Dessa forma, muitos deles não procuram serviço médico e muito menos, necessitam de internação hospitalar. Podemos afirmar que as pneumonias necessitem de maior número de leitos hospitalares do que a asma, por exemplo. Mas os dados não indicam que as pneumonias são um grupo mais prevalente do que a asma na população.

Dentre as doenças respiratórias crônicas, a asma e as doenças pulmonares obstrutivas crônicas (DPOC) destacam-se como importantes causas de morbimortalidade. De fato, a asma é uma das causas mais comuns de doença crônica em crianças e adultos ${ }^{3}$. Sua prevalência vêm aumentando na faixa etária pediátrica. Porém, segundo Phelan $^{28}$, não há um consenso mundialmente aceito na definição de asma em crianças. Dessa forma, muitos estudos que se propõem em mostrar o aumento da prevalência usam diferentes métodos de medidas e de análises, dificultando a comparação entre eles.

Nossos dados relacionados a asma mostraram uma baixa morbi-mortalidade entre os pacientes internados, com incidência maior na população menor de 5 anos. De 1995 a 2000, houve ligeira tendência geral de aumento da taxa de internações com resultados não estatisticamente significantes. Restringindo ao estudo por faixas etárias, observamos que as internações de pacientes com idade menor de 5 anos obtiveram aumento estatisticamente significante no período estudado. A educação ambiental e maior cobertura vacinal são fatores que podem diminuir a incidência de asma, principalmente em crianças. Isto porque a infecção de vias aéreas superiores, pêlo de animais, ácaros, uso de ácido acetil-salicílico e tabagismo são ou podem ser importantes desencadeadores de crises asmáticas e muitos deles podem ser prevenidos ${ }^{3,25}$. Devemos também relembrar que estamos analisandos essas doenças quanto a severidade das crises e não, quanto a gravidade da asma.

Diette et al. ${ }^{7}$, em estudo de coorte com 6590 pacientes, observaram que as taxas de hospitalizações e de morte em pacientes com asma nos Estados Unidos eram maiores em pacientes com idade superior a 64 anos do que em pacientes mais jovens. Porém, a idade cronológica não era um risco independente para hospitalizações, tendo em vista o maior número de comorbidades nessa faixa etária. Em vários estudos, os fatores de risco independentes que estavam associados a hospitalizações por asma eram: o sexo feminino, a raça não branca, o menor nível educacional, as piores condições físicas e a maior freqüência de sintomas da asma ${ }^{4,7,10}$.

A asma é incomum causa de mortalidade, uma vez que é tratável e controlável ${ }^{1}$. Mesmo assim, alguns países vêm reportando aumento na mortalidade por asma $^{3,4}$.

Após o crescimento da mortalidade, principalmente entre os jovens, visto na década de 80 em vários países, aumentou-se o interesse no padrão epidemiológico, a preocupação com a eficácia dos tratamentos e surgiram manuais para diagnóstico e controle da asma ${ }^{37,38}$.

A tendência da mortalidade nos Estados Unidos (EUA) no início do século mostrou-se estável, havendo um aumento em 1940 e 1950, seguido de decréscimo até 1980. A partir de 1978 parece haver um acréscimo ${ }^{22,37}$. A mortalidade entre 1980 e 1997 cresceu 31\% apesar do avanço no tratamento ${ }^{4}$. No Reino Unido, estatísticas admissionais e documentos sugerem aumento da mortalidade entre jovens ${ }^{1}$.

Essa tendência crescente não surgiu apenas nos EUA como também no Canadá, Inglaterra e País de Gales, Nova Zelândia e Japão. A Austrália demonstrou crescimento seguido de decréscimo, a Alemanha, decréscimo, assim como a Holanda, principalmente entre a faixa etária dos 5 aos $65 \operatorname{anos}^{37}$.

São Paulo, em estudo de 1960, comparado com Ribeirão Preto, Bristol (Reino Unido), São Francisco (EUA) e mais oito cidades da América Latina, obteve a menor taxa de mortalidade. A utilização de cuidados médicos cresceu em São Paulo a partir da segunda metade de 1980, levando a programas intervencionistas, com consequiente melhora do tratamento, havendo declínio da mortalidade entre 1970 e 1992 em SP 22 .

A mortalidade por asma mostrou crescimento pouco significante no período aqui estudado. Ao contrário das outras Doenças Respiratórias, as mulheres têm taxa de mortalidade mais elevadas que homens em todas as faixas etárias. Enquanto as taxas entre homens apontaram tendência à queda, as mulheres tiveram leve crescimento (apesar da pouca significância estatística de ambas). Há tendência decrescente para menores que 5 anos e crescente para faixa intermediária e idosos, também sem significância.

Para DPOC, o envelhecimento da população e o maior tempo de exposição ao tabagismo com o passar dos anos, são fatores que podem explicar o aumento das taxas de internação na faixa etária contendo a população com idade maior que 65 anos. Segundo página sobre o Censo Demográfico 2000 no site do IBGE, na composição da população por idade, o grupo de idosos de 65 anos ou mais, por sua vez, seguiu trajetória ascendente: 4,83\%, em 1991, contra 5,85\%, em 2000, indicando envelhecimento da população. A idade mediana da população brasileira (idade que separa os $50 \%$ mais jovens dos $50 \%$ mais velhos) aumentou dois anos e meio em nove anos, alcançando, em 2000, o patamar dos 24,2 anos, sendo de 23,5 anos para os homens e 24,9 anos para as mulheres. A idade mediana em 1991 era 21,7 anos ${ }^{18}$. Além disso, pudemos notar aumento do tempo de hospitalização e da média de idade dos pacientes internados por DPOC. A 
mortalidade também foi maior nestes pacientes. A DPOC é a quinta causa de morte nos EUA, é a doença de base mais comum em pacientes com pneumonia de comunidade necessitando freqüentemente de hospitalização ${ }^{24,26}$. Em alguns países como a Holanda ${ }^{36}$, a mortalidade por DPOC vem aumentando entre os idosos.

A DPOC é uma grande causa de morbi-mortalidade no mundo. Está se tornando cada vez mais prevalente em mulheres de vários países, tais como Índia, México, Cuba, Egito, África do Sul e China ${ }^{21}$. Em média, aproximadamente 5 a $15 \%$ dos adultos em países industrializados têm DPOC definida pela espirometria. Em 1990 , a DPOC era considerada a $12^{\text {a }}$. causa de morbimortalidade e espera-se que possa se tornar a $5^{\text {a }}$. causa por volta de 2020 ou a $3^{\text {a }}$. causa de mortalidade isolada no mundo ${ }^{2,36}$.

O maior fator de risco ambiental para o desenvolvimento da DPOC é o tabagismo ${ }^{21,23}$. Em não fumantes, o volume expiratório forçado em 1 segundo (VEF1) declina a uma taxa média de aproximadamente 20 a $30 \mathrm{~mL}$ por ano durante a vida adulta. $\mathrm{Na}$ maioria dos fumantes, a taxa média de declínio é aumentada a 30-45 $\mathrm{mL}$ por ano, mas no subconjunto de pacientes com predisposição genética a desenvolver DPOC, a taxa de declínio chega a 80-100 mL por ano. A deficiência de a1antitripsina é o único fator de risco genético comprovado para o desenvolvimento da DPOC. Há evidência de uma relação entre a gravidade da doença pulmonar e o número de cigarros-anos fumados 21 . Fatores ambientais, como poluição do ar e exposição a antígenos ocupacionais, também têm importante papel na patogênese ${ }^{21,23}$.

As pneumonias são a sexta causa mais importante de morte nos EUA, atrás da doença cardíaca, câncer, doença vascular cerebral, lesões não intencionais e doença pulmonar obstrutiva crônica (DPOC). A mudança na epidemiologia dos agentes etiológicos da pneumonia, o aumento na ocorrência de resistência a antimicrobianos, o surgimento de novos patógenos nos EUA alertou para o crescimento da importância em Saúde Pública das Doenças Respiratórias Agudas ${ }^{24}$.

As pneumonias são o principal diagnóstico de infecção respiratória aguda, sendo responsáveis por um terço das mortes em menores que cinco anos em países em desenvolvimento ${ }^{13,31}$. Podem-se citar fatores de risco como aglomeração domiciliar, a freqüência em creches, fumo dos pais, poluição ambiental, índices como renda familiar, instrução da mãe, variáveis tais quais peso ao nascer, desnutrição e desmame precoce ${ }^{8}$. A taxa de mortalidade vem caindo ao longo dos anos exceto entre os idosos ${ }^{15}$.

Nos EUA, de 1979 a 1994, a taxa de mortalidade para pneumonias e para gripe cresceu 59\%. A taxa ajustada para idade (utilizando-se a população base de 1980) cresceu $22 \%$. No período, também houve aumento de $44 \%$ para a faixa etária de maiores que 65 anos, notando-se também aumento na faixa entre 20 a 44 anos. Esse crescimento recente na mortalidade reflete tanto o crescimento da população idosa quanto também o maior acometimento dessa faixa etária ${ }^{24}$. A pneumonia é mais comum e crescente entre faixas etárias de idosos e pacientes com comorbidades, além disso, pacientes idosos têm risco relativo de morte maior quando comparado com os mais jovens com pneumonia ${ }^{37}$.

Nossos dados revelaram um aumento do número de internações por pneumonias no primeiro semestre e queda, no decorrer do segundo. Os meses com maior número de internações são os mais relacionados ao inverno e aqueles com menor número são os mais relacionados com o verão.

A maioria dos estudos que mostra o padrão sazonal se localiza em países de clima temperado, em que as estações do ano são mais definidas. Na Finlândia, por exemplo, as grandes diferenças climáticas entre verão e inverno têm importante papel no padrão sazonal das hospitalizações por DPOC e pneumonias, com maior número de internações durante o inverno ${ }^{34,35}$.

Para a asma, observamos flutuação no número de internações no decorrer dos meses, com picos principalmente entre março e maio (meses de outono) e mínimo em janeiro (verão). Vários estudos demonstraram a mesma tendência, principalmente na faixa etária pediátrica ${ }^{6,17,19}$. Na Grécia e em Malta (países mediterrâneos), foram descritos variações sazonais associadas ao tempo frio e úmido, com diferenças entre subgrupos etários na infância ${ }^{16,29}$. A mortalidade por DPOC apresenta caráter oscilatório e gráfico crescente. A asma também apresenta a oscilação parecendo ter leve aumento ao longo do período apesar da grande variabilidade. A curva das pneumonias vem diminuindo ao longo do tempo, principalmente a partir de 1995 . Os números permanecem altos durante os meses de verão, apresentando importante aumento nos meses de inverno.

A evolução das Doenças Respiratórias ao longo do período mostra que o número de casos de morte aumenta durante os meses de inverno e diminui nos meses de verão. Doenças Respiratórias totais mostram variação durante os meses do ano, com crescimento das taxas durante o inverno e queda no verão. Todavia, DPOC não mostra tal variação. O aumento da mortalidade durante os meses de inverno é um fato no mundo industrializado. A maioria ocorre por doenças respiratórias e estas influem também nas cardiovasculares $^{5,15}$.

A média de temperatura, a umidade do ar e poluição ambiental são fatores considerados importantes na sazonalidade das doenças respiratórias ${ }^{11}$. A temperatura deve ser uma das variáveis climáticas que age sinergisticamente com outras, promovendo a difusão das Doenças Respiratórias. Partículas em suspensão na atmosfera podem criar ambiente favorável para a transmissão de microorganismos, o que é visto em vários estudos com associação entre poluição ambiental e mortalidade por Doenças Respiratórias ${ }^{3,21}$.

Deve-se ressaltar que houve alteração da Classificação Internacional de Doenças (CID) durante o período estudado. Isso não configura uma limitação ao 
estudo em si, mas talvez ao preenchimento do diagnóstico da doença pelos profissionais da saúde. Segundo o Centro Brasileiro de Classificação de Doença da Faculdade de Saúde Pública da Universidade de São Paulo (comunicação pessoal), a categoria mais afetada pela mudança do CID foi o grupo das pneumonias.

\section{CONCLUSÕES}

Nesse trabalho, pudemos observar uma tendência não estatisticamente significante de diminuição das taxas de internação por doenças respiratórias no período de 1995 a 2000, no município de São Paulo. Essa tendência foi mais marcante entre a população do sexo masculino, que apresentou resultados estatisticamente significantes. As taxas de mortalidade decresceram (apesar de pouco significativo) entre 1991 e 2000. Os homens representaram a maior parcela, sendo em média responsáveis por $56 \%$ das mortes, havendo uma queda estatisticamente significante entre eles. Entre os menores que 65 anos, houve um decréscimo real da taxa no período estudado, enquanto os idosos apresentaram uma tendência crescente, como já vista em outros países ${ }^{38}$.

Nossas análises revelaram a participação importante das pneumonias no total de doenças respiratórias, representando cerca de $47 \%$ das internações e $61 \%$ das mortes. Em relação a tendência no período, também observamos uma redução nas taxas de internações, principalmente para o sexo masculino. Fatores como maior cobertura vacinal e melhoria do sistema de saúde são fatores que contribuiriam para a diminuição mais acentuada da incidência das pneumonias em nosso meio. É importante causa de mortalidade, refletindo suas tendências nas curvas totais. A pneumonia para a faixa etária I representou cerca de $80 \%$ das mortes por Doenças Respiratórias. Em todas as faixas etárias tenderam à queda no período observado, padrão já demonstrado em outro estudo ${ }^{22}$ para o período de 1970 a 1992 no Estado de São Paulo.

A mortalidade atinge mais aos homens que às mulheres pela pneumonia. Como as outras doenças, os idosos são a faixa mais atingida. Mas, diferentemente, em seguida vem os menores que 5 anos. No período, observouse um decréscimo entre todas as faixas etárias (só não estatisticamente significante para os maiores que 65 anos) não visto em estudos de outros países, EUA ${ }^{24}$, por exemplo.

Como vimos, as doenças agudas, como as pneumonias, vêm apresentando uma tendência a diminuir em incidência. Ao contrário de doenças crônicas como a DPOC, que apresentou aumento das taxas de internação, principalmente em faixas etárias mais avançadas. A mortalidade por DPOC é a segunda em importância, crescente entre os mais velhos e também atingem mais aos homens.

De maneira geral, estes resultados indicam uma ligeira mudança no perfil destas doenças no nosso meio (Brasil), o que traz conseqüências imediatas para o planejamento de serviços e para a formulação de políticas de saúde.

Toyoshima MTK, Munemasa G, Gouveia N. Temporal trends in morbidity and mortality for respiratory diseases in São Paulo, Brazil. Rev Med (São Paulo) 2003 jan.-dez.;82(1-4):67-77.

\begin{abstract}
S: Respiratory diseases are important causes of morbi-mortality in all agegroups, with considerable economic and social impacts. Several studies have been poiting out an increase in hospitalizations and deaths due to respiratory diseases. However, few studies have analysed such recent trends in Brazil. In the present study we analyzed hospitalizations occuring from 1995 to 2000 and mortality from 1991 to 2000 through descriptive tables and graphs, correlation analysis and trend tests. We observed a decrease in the rate of hospitalizations for respiratory diseases between 1995-1997 and an increase between 1997-2000 for all agegroups. Increasing trends were also observed for mortality between 1991-2000. Specific patterns for different causes of respiratory diseases, different agegroups and sexes were observed. We concluded that, in the period studied, acute respiratory diseases such as pneumonia had a greater contribution to the respiratory morbi-mortality but with a decreasing trend. On the other hand, chronic respiratory diseases have presented increasing trends in the period. These results point out to a changing pattern in the respiratory diseases morbi-mortality with direct consequences for public health policy making.
\end{abstract}

KEYWORDS: Sickness impact profile. Comorbidity. Respiratory diseases/mortality. Indicators of morbidity and mortality. Seasonal variations. Time factors.

\title{
REFERÊNCIAS
}

1. Anderson HR. Is the prevalence of asthma changing? Arch Dis Chil 1989;64:172-5.

2. Anto JM, Vermeire P, Vestbo J, Sunyer J. Epidemiology of chronic obstructive pulmonary disease. Eur Respir J 2001;17:982-94.

3. Burney PGJ. Strategy for asthma. BMJ Br Med J 1991;303:571-3.
4. Carr W, Zeitel L, Weiss, K. Variations in Asthma Hospitalizations and Deaths in New York City. Am J Public Health 1992;82:59-65.

5. Crombie DL, Fleming DM, Lancashire RJ, Cross KW. Concurrence of monthly variations of mortality related to underlying cause in Europe. J Epidemiol Community Health 1995:49:373-8. 
6. Dales RE, Schweitzer I, Toogood JH, Drouin M, Yang W, Ddovich J et al. Respiratory infections and the autumn increase in asthma morbidity. Eur Respir J 1996;9:72-7.

7. Diette GB, Krishnan JA, Dominici F, Haponik E, Skinner EA. Asthma in older patients: factors associated with hospitalization. Arch Intern Med 2002;162:1123-32.

8. Duchiade MP, Beltrão KI. Mortalidade infantil por causas na região metropolitana do Rio de Janeiro, 1976-1986: associação com variáveis socioeconômicas, climáticas e ligadas à poluição do ar. Rev Bras Estudos Pop 1992;9:11537.

9. Duchiade MP. Poluição do ar e doenças respiratórias: uma revisão. Cad Saúde Publ 1992;8:311-30.

10. Eisner MD, Katz PP, Yelin EH, Shiboski SC, Blanc PD. Risk factors for hospitalization among adults with asthma: the influence of sociodemographic factors and asthma severity. Respir Res 2001;2:53-60.

11. Fleming DM, Cross KW, Crombie DL, Lancashire RJ. Respiratory illness and mortality in England and Wales. Eur J Epidemiol 1993;9:571-6.

12. Fundação Sistema Estadual de Análise de Dados (Fundação SEADE). Informações dos municípios paulistas (São Paulo em dados). Disponível em: http://www.seade.gov.br/cgi-bin/ homev98/seade_op.ksh.

13. Garenne M, Ronsmans C, Campbell H. The magnitude of mortality from acute respiratory infections in children under 5 years in developing countries. World Health Stat Q 1992;45:180-91.

14. Gouveia N, Fletcher T. Respiratory diseases in children and outdoor air pollution in São Paulo, Brazil: a time series analysis. Occup Environ Med 2000;57:477-82.

15. Graham N. The epidemiology of acute respiratory infections in children and adults: a global perspective. Epidemiol Rev 1990;12:149-78.

16. Grech V, Balzan M, Asciak RP, Buhagiar A. Seasonal variations in hospital admissions for asthma in Malta. J Asthma 2002;39:263-8.

17. Harju T, Keistinen T, Tuuponen T, Kivela SL. Seasonal variation in childhood asthma hospitalisations in Finland, 1972-1992. Eur J Pediatr 1997;156:436-9.

18. Instituto Brasileiro de Geografia e Estatística (IBGE). Censo demográfico 2000. Disponível em: http://www.ibge.gov.br/ censo/default.php.

19. Khot A, Burn R, Evans N, Lenney C, Lenney W. Seasonal variation and time trends in childhood asthma in England and Wales 1975-81. Br Med J (Clin Res Ed) 1984;289:2357.

20. Kunst AE, Looman CWN, Mackenbach JP. The decline in winter excess mortality in the Netherlands. Int J Epidemiol. 20:971-7.

21. Lomas DA, Silverman EK. The genetics of chronic obstructive pulmonary disease. Respir Res 2001;2:20-6.

22. Lotufo PA, Benseñor IJM, Lolio CA. Mortality from asthma in the state of S. Paulo, Brazil (1970-1992). Rev. Saude Publica, 1995;29:434-9.
23. Mannino DM. COPD: epidemiology, prevalence, morbidity and mortality, and disease heterogeneity. Chest 2002;121(5 Suppl):121S-126S.

24. Pneumonia and influenza death rates - United States, 19791994. MMWR Morb Mortal Wkly Rep;44(28):535-7, 1995.

25. National Institutes of Health. Global initiative for asthma Pocket Guide for Asthma Management and Prevention. 1998.

26. Organização Mundial de Saúde. Classificação Internacional de Doenças - Revisão 1975. Centro da OMS para Classificação de Doenças em Português. Brasília: Ministério da Saúde/Universidade de São Paulo; 1985.

27. Organização Mundial de Saúde. CID-10/ Organização Mundial de Saúde; tradução - Centro Colaborador da OMS para a Classificação de Doenças em Português. 3a ed. São Paulo: Editora da Universidade de São Paulo; 1996.

28. Phelan PD. Asthma in children: epidemiology. BMJ 1994;308:1584-5.

29. Priftis K, Anagnostakis J, Harokopos E, Orfanou I, Petraki M, Safoni-Papageorgiou P. Time trends and seasonal variation in hospital admissions for childhood asthma in the Athens region of Greece: 1978-88. Thorax 1993;48(11):1168-9.

30. PROAIM. Informe técnico $n^{\circ} 1$. Sec. Municipal de Saúde PMSP, 1991, mimeo.

31.Rello J, Rodriguez R, Jubert P, Alvarez B. Severe community acquired Pneumonia in the elderly: epidemiology and prognosis. Clin Infect Dis 1996;23:723-8.

32. Ribeiro TM, Guedes JS, et al. Importância das infecções respiratórias agudas em crianças no Município de São Paulo. Rev Paul Pediatr 1985.

33. Saynajakangas P, Keistinen T, Tuuponen T. Seasonal fluctuations in hospitalisation for pneumonia in Finland. Int J Circumpolar Health 2001;60:34-40.

34. Brasil. Ministério da Saúde - Sistema Único de Saúde - . Sistema de informações hospitalares do SUS (SIH/SUS). Disponível em: http://tabnet.datasus.gov.br/cgi/ deftohtm.exe?sih/cnv/mrsp.def.

35. Vilkman S, Keistinen T, Tuuponen T, Kivela SL. Seasonal variation in hospital admissions for chronic obstructive pulmonary disease in Finland. Arctic Med Res 1996;55:1826.

36. Ward SA, Casaburi R. $21^{\text {st }}$ Century perspective on chronic obstructive pulmonary disease. Respiration 2001;68:557-61.

37. Weiss KB, Gergen PJ, Wegener DK. Breathing better or wheezing worse? The changing epidemiology of asthma morbidity and mortality. Annu Rev Publ Health 1993;14:491513.

38. Wever-Hess J, Wever AMJ. Asthma statistics in the Netherlands 1980-1994. Respir Med 1997;91:417-22.

39. World Health Organization. 1995: The Division of Diarrhoeal and Acute Respiratory Disease Control. Available from: URL:http://www.who.int/chd/publications/cdd/ pofact77.htm.

40. World Health Organization. 1997: World Health Report. Geneva: WHO; 1998. 